\title{
New lidocaine lozenge as topical anesthesia compared to lidocaine viscous oral solution before upper gastrointestinal endoscopy
}

This article was published in the following Dove Press journal:

Local and Regional Anesthesia

30 May 2012

Number of times this article has been viewed

\author{
Stine Mogensen' \\ Charlotte Treldal' \\ Erik Feldager ${ }^{2}$ \\ Sylvia Pulis' \\ Jette Jacobsen ${ }^{3}$ \\ Ove Andersen' \\ Mette Rasmussen ${ }^{4}$ \\ 'Clinical Research Centre, \\ Hvidovre University Hospital, \\ Copenhagen, Denmark; ${ }^{2}$ Department \\ of Gastroenterology, Hvidovre \\ University Hospital, Copenhagen, \\ Denmark; ${ }^{3}$ Department of \\ Pharmaceutics and Analytical \\ Chemistry, Faculty of Pharmaceutical \\ Sciences, University of Copenhagen, \\ Denmark; ${ }^{4}$ Department of \\ Pharmacology and Pharmacotherapy, \\ Faculty of Pharmaceutical Sciences, \\ University of Copenhagen, Denmark
}

Correspondence: Charlotte Treldal Clinical Research Centre, Hvidovre University Hospital, Kettegaard Allé 30, DK-2650 Hvidovre, Denmark

Tel +4538626077

Fax +45 38623797

Email sugetablet@gmail.com
Objective: To evaluate the effect and acceptance of a new lidocaine lozenge compared with a lidocaine viscous oral solution as a pharyngeal anesthetic before upper gastrointestinal endoscopy (UGE), a diagnostic procedure commonly performed worldwide during which many patients experience severe discomfort mostly because of the gag reflex.

Participants: The single-blinded, randomized, controlled study involved 110 adult patients undergoing diagnostic UGE at the Department of Gastroenterology, Hvidovre University Hospital, Denmark.

Methods: The patients were randomized to receive either $100 \mathrm{mg}$ lidocaine as a lozenge or $5 \mathrm{~mL}$ lidocaine viscous oral solution $2 \%$. Intravenous midazolam was administered if needed. The effect of a lidocaine lozenge in reducing patient discomfort, including the gag reflex, during UGE compared with a lidocaine oral solution was assessed.

Results: Questionnaires from the patients showed that the gag reflex was acceptable for $64 \%$ in the lozenge group compared with $33 \%$ in the oral solution group $(P=0.0072)$. UGE was evaluated as acceptable by $69 \%$ in the lozenge group compared with $39 \%$ in the oral solution group $(P=0.0092)$. The taste was evaluated as good by $78 \%$ in the lozenge group $(P<0.0001)$, and $82 \%$ found the lozenge to have good texture $(P<0.0001)$.

Conclusion: The lozenge reduced the gag reflex, diminished patients' discomfort during UGE, and was evaluated as having a good taste and texture. The lozenge improved patients' acceptance of UGE.

Keywords: upper gastrointestinal endoscopy, topical pharyngeal anesthetic, lidocaine lozenge

\section{Introduction}

Upper gastrointestinal endoscopy (UGE) is a common diagnostic procedure performed throughout the world. ${ }^{1}$ Many patients experience severe discomfort during UGE, mostly because of the strong gag reflex that occurs when the endoscope is passed through the pharynx. ${ }^{2}$

To ease patient discomfort during UGE, topical pharyngeal anesthesia is often used, either alone or combined with intravenous (IV) sedation. ${ }^{3}$ As a topical pharyngeal anesthesia, lidocaine is the primary choice of drug, often administered as either a spray or a viscous solution. The spray device has a tendency to provoke the gag reflex, and the solution is unpleasant to swallow because of its viscous texture and bitter taste. The bitter taste of lidocaine can decrease patient acceptance of UGE. ${ }^{3,4}$

The hypothesis of this study was that a new lidocaine lozenge could increase patient acceptance of UGE. It was anticipated that the lozenge would reduce the gag reflex 
during the procedure, and make the taste and texture of the local anesthetic formulation more acceptable.

The aim of this study was to evaluate the effect and patient acceptance of the new lidocaine lozenge compared with a lidocaine viscous solution as a topical pharyngeal anesthetic before UGE.

\section{Methods}

\section{Lidocaine lozenge formulation}

The lozenges for this study were manufactured at the Pharmacy of the Capital Region, Denmark. The lozenges were prepared using lidocaine hydrochloride as the active compound. A sweetening agent, a glidant, and a binder were added. The bitter taste of lidocaine was disguised with liquorice powder. The lozenges were formulated to release lidocaine to the pharynx over 10 minutes.

\section{Lidocaine viscous oral solution}

Lidocaine viscous oral solution $2 \%(\mathrm{w} / \mathrm{w})$ with liquorice flavor was used as the standard topical anesthesia before UGE at Hvidovre University Hospital. The oral solution was manufactured at the Pharmacy of the Capital Region, Denmark.

\section{Patients}

Consecutive patients undergoing UGE at Hvidovre University Hospital were eligible to participate. The inclusion criteria were age between 18 and 80 years, use of safe contraception for a minimum of three months before undergoing UGE (applied only to fertile women); the ability to speak, read, and understand Danish; and the ability to provide oral and written consent. The exclusion criteria were known allergy to lidocaine, history of dry mouth, pregnancy, or breastfeeding. Lidocaine is contraindicated in patients with severe liver impairment; however, no such patients were enrolled in the study because they are not treated ambulant.

\section{Study design}

The study was a single-blinded, randomized, controlled study and conducted as an as-treated analysis. The study was approved by the Regional Committee on Biomedical Research Ethics, Hillerød, Denmark, the Danish Medicine Agency, and the Danish Data Protection Agency. The Good Clinical Practice (GCP) unit of Copenhagen University Hospital monitored the data throughout the study. The study was registered at the public database ClinicalTrials.gov (Jour No NCT00791024) and was performed in accordance with the Helsinki Declaration and national ethical rules for biomedical research.

\section{Procedure}

An IV cannula was placed on the back of the patient's hand according to standard procedure. Before and during the UGE procedure, the patient's blood pressure, heart rate, and oxygen saturation were monitored. A research assistant carried out the randomization by opening a sealed opaque envelope. Afterwards, a research assistant administered pharyngeal anesthesia to the patient in the absence of the endoscopist, who was blinded to the randomization. To ensure blinding, the patients and nurses were advised to avoid any unnecessary discussion of the pharyngeal anesthesia after administration. The patients received $100 \mathrm{mg}$ lidocaine either as the lozenge or as $5 \mathrm{~mL}$ of the viscous oral solution. The lozenge was to be sucked until it was completely dissolved, whereas the solution was to be swallowed. Ten minutes after the pharyngeal anesthesia was administered, the patients were ready for UGE.

For awake sedation, IV midazolam (1.25-5 mg) was administered either before or during the procedure if necessary. A research assistant noted if IV midazolam was given as well as the time needed for the passage of the endoscope through the pharynx and the time for the complete UGE.

Fifteen endoscopists, who had experience with more than 500 UGEs, performed the procedure with a video esophagogastroduodenoscope (Olympus, Woodstock, NY).

\section{Patient assessment}

After the UGE and when fully alert, the patient evaluated the effect of the local anesthetic by completing a questionnaire. The questions included patients' perception of the taste and texture of the lozenge or solution, the effect of the local anesthetic, and acceptance of the gag reflex. The discomfort during the procedure was assessed on a scale from 0 to 10 , where 0 was no discomfort and 10 was the worst discomfort imaginable, and a 4-point scale where $1=$ no discomfort, 2 = slight discomfort, 3 = moderate discomfort, and $4=$ severe discomfort.

\section{Endoscopist assessment}

Before the UGE, the endoscopist rated the expected difficulty of the UGE on a 4-point scale as follows: 1 = very easy, 2 = easy, $3=$ difficult, and $4=$ very difficult. The same scale was used after the procedure when the endoscopist evaluated the complete impression of the UGE.

\section{Statistical analysis}

The sample size was determined with the following data: a minimum relevant clinical difference of 1.50 on a 
visual analogue scale of discomfort, an expected standard derivation of 2.00, a significance level of 0.05 , and a power of $80 \%$. This led to a sample size of 50 patients in each of the two groups, and 110 patients were included to account for dropouts.

Categorical variables between the lozenge group (L group) and the solution group (S group) were compared using the chi-square test. Differences between the two groups for continuous variables were analyzed using a two-sample Student's $t$-test. The level of statistical significance was defined as a $P$ value $<0.05$. All statistical analyses were performed with the SAS statistical software (v9.1; SAS Institute Inc, Cary, NC).

\section{Results}

During eight weeks, 298 consecutive patients were screened, of whom 110 fulfilled the inclusion criteria and were included. The primary reasons for not including patients were age $(27 \%)$ or nonattendance/cancellation of UGE $(26 \%)$. The patients were enrolled and randomized to the study arms, with 55 patients randomized to the L group and 55 patients to the $\mathrm{S}$ group. Six patients from the L group and four from the $\mathrm{S}$ group were withdrawn, either because of amnesia caused by sedation (six patients), suspension of UGE (two patients), change in endoscopic procedure (one patient), or unwillingness to answer the questionnaire (one patient). A total of 49 patients from the $\mathrm{L}$ group and 51 patients from the $\mathrm{S}$ group completed the study and were included in the analyses. The demographic data for the two treatment groups were similar with regard to age, sex, body mass index, the American Society of Anesthesiologists physical status classification system, and the number of patients in each group who had experienced an UGE before, as shown in Table 1.

Table I Demographic data of enrolled patients

\begin{tabular}{|c|c|c|}
\hline & L group $(n=49)$ & $S$ group $(n=51)$ \\
\hline Mean age $\pm S D$, years & $54.2 \pm 14.3$ & $50.6 \pm 15.8$ \\
\hline \multicolumn{3}{|l|}{ Sex, \% (n) } \\
\hline Male & $47(23)$ & $45(23)$ \\
\hline Female & $53(26)$ & $55(28)$ \\
\hline Mean $\mathrm{BMI} \pm \mathrm{SD}, \mathrm{kg} / \mathrm{m}^{2}$ & $28.8 \pm 7.4$ & $29.4 \pm 8.4$ \\
\hline \multicolumn{3}{|l|}{ ASA physical status, \% (n) } \\
\hline I & $43(2 I)$ & $43(22)$ \\
\hline II & $53(26)$ & $55(28)$ \\
\hline III & $4(2)$ & $2(I)$ \\
\hline Tried UGE before, \% (n) & $65(32)$ & $67(34)$ \\
\hline
\end{tabular}

Abbreviations: SD, standard deviation; BMI, body mass index; ASA, American Society of Anesthesiologists; UGE, upper gastrointestinal endoscopy; L group, lozenge group; $S$ group, solution group.

Note: $P=0.0072$.

\section{Patient assessment}

The patient assessment was done either shortly after the procedure for nonsedated patients ( 52 patients) or after full recovery from sedation (48 patients). There was no significant difference between the number of patients receiving sedation in the two groups $(P=0.16)$.

The evaluation of the acceptance of the discomfort showed that more patients in the L group found the discomfort acceptable (59\% versus 39\%) and more patients in the $\mathrm{S}$ group found the discomfort unacceptable (12\% versus $6 \%$ ). The results were significantly different between the two groups $(P=0.046)$.

The acceptance of the gag reflex during UGE was also significantly different $(P=0.0072)$, with more patients in the $\mathrm{L}$ group finding the gag reflex acceptable $(64 \%$ versus $33 \%$; see Figure 1). The patient assessment of the acceptance of UGE showed that more patients in the L group found the procedure acceptable compared with the S group (69\% versus $39 \%, P=0.0092$; see Figure 2). A subgroup analysis of the results from the nonsedated patients shows that more patients in the L group found the gag reflex acceptable (55\% versus $22 \%, P=0.04)$ as well as UGE acceptable (76\% versus $39 \%$, $P=0.02$ ) compared with the patients in the $\mathrm{S}$ group.

The majority of patients in the $\mathrm{L}$ group evaluated taste (78\% versus $16 \%, P<0.0001$; see Figure 3 ) and texture (82\% versus $29 \%, P<0.0001)$ as good compared with the $\mathrm{S}$ group (Figure 4).

None of the patients experienced any adverse events.

\section{Endoscopist assessment}

Most endoscopists graded the introduction of the endoscope and passage through the pharynx as either very easy or easy, and there was no significant difference between the two

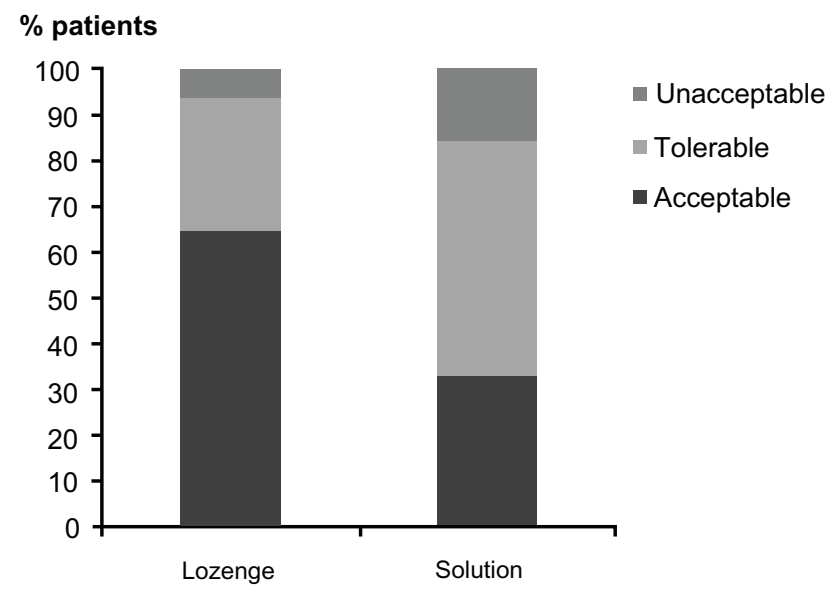

Figure I Patient acceptance of the gag reflex during upper gastrointestinal endoscopy 


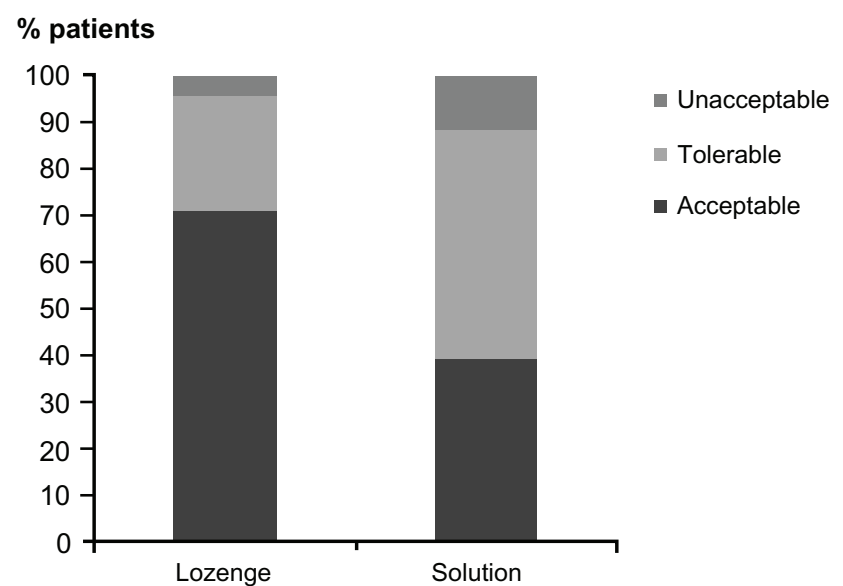

Figure 2 Patient acceptance of upper gastrointestinal endoscopy. Note: $P=0.0092$.

groups $(P=0.54)$. Time for the passage of the endoscope through the pharynx as well as the total time for the UGE was similar for the groups $\left(P_{\text {pharynx }}=0.53\right.$ and $\left.P_{\text {total }}=0.41\right)$.

Before the UGE, the endoscopists assessed their expectation of the procedure after a consultation with the patient. After the UGE, the endoscopist assessed the procedure again. For both assessments, there was no significant difference $\left(P_{\text {before }}=0.67\right.$ and $\left.P_{\text {after }}=0.57\right)$ between the two groups.

\section{Discussion}

The study is the first study evaluating the overall performance/ acceptance of the lidocaine solution versus the lozenge taking into account the taste of the solution. We are not aware of any other trials showing a higher degree of acceptance.

A few other studies have evaluated the use of a lozenge as topical anesthesia before UGE. ${ }^{3,5,6}$ However, these results are

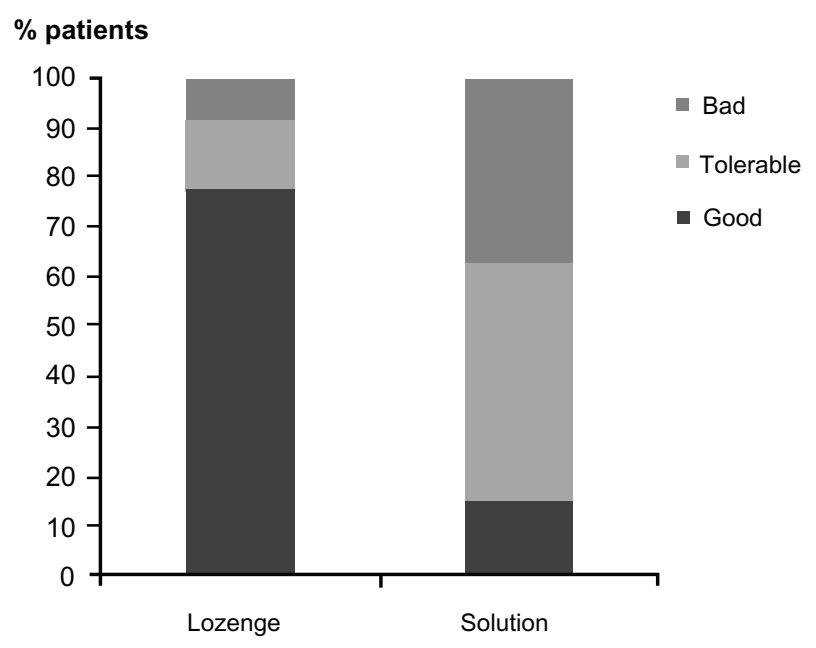

Figure 3 Patient assessment of the taste of the lozenge or solution. Note: $P<0.0001$.

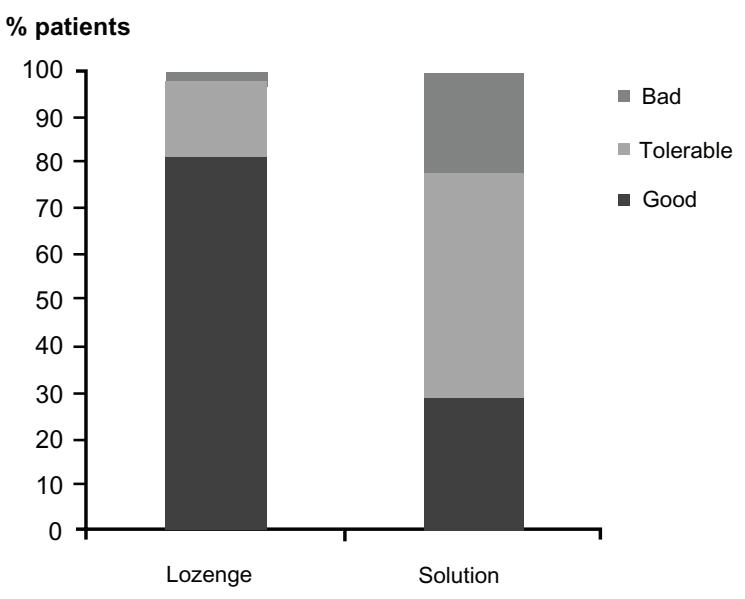

Figure 4 Patient assessment of the texture of the lozenge or solution. Note: $P<0.0001$.

not directly comparable with the results in this study because other active drugs or different doses of lidocaine were used. Heuberger et $\mathrm{al}^{6}$ randomly evaluated the same dose of anesthetic (20 mg oxybuprocaine) given by a spray or by a lozenge in 132 consecutive patients. Heuberger et al found patient acceptance was significantly better when topical anesthesia was administered as a spray than with a lozenge. ${ }^{7}$ Shaoul et $\mathrm{al}^{3}$ randomly evaluated whether a benzocaine/tyrothricin (3.5 $\mathrm{mg}$ benzocaine and $2 \mathrm{mg}$ tyrothricin) lozenge with conscious sedation was superior to conscious sedation alone in 174 patients undergoing UGE. Shaoul et al concluded that the use of the lozenge with conscious sedation had no advantages over conscious sedation alone. ${ }^{3}$ Both studies used drugs other than lidocaine, where oxybuprocaine is more potent and benzocaine is less potent than lidocaine. ${ }^{8}$ Furthermore, the patients in both studies also received sedation, which likely biases the assessment of the effects of topical anesthesia as sedation induces antegrade amnesia. ${ }^{9}$ A recent study by Canon et $\mathrm{al}^{5}$ compared the effectiveness of a lidocaine spray with a lozenge in a randomized placebo-controlled study in 191 unsedated consecutive patients. ${ }^{5}$ The lozenge was found to have a better taste than the spray, but overall the lidocaine spray was shown to be superior to the lozenge. The superiority of the spray group may be attributed to the considerable difference in total dose as the lozenge group received only $20 \mathrm{mg}$ of lidocaine whereas the spray group received $60 \mathrm{mg}$ of lidocaine. ${ }^{5}$

The discomfort associated with UGE is primarily caused by a strong gag reflex. ${ }^{2,10}$ In this study, twice as many patients in the L group found the gag reflex acceptable compared with those in the $\mathrm{S}$ group. This could indicate that the lozenge has a better anesthetic effect, thereby reducing patient discomfort during UGE. This finding is supported by 
the assessment of the discomfort, which showed that more patients in the L group found the discomfort acceptable compared with those in the $\mathrm{S}$ group. Because the results for patient acceptance of UGE showed that more patients in the L group found UGE acceptable, this could indicate that patient acceptance of UGE is affected by the discomfort. This finding indicates that the lozenge improves patient acceptance of UGE. This finding is consistent with a study by Amornyotin et $\mathrm{al}^{11}$ showing that comfort is an important predictor of patient acceptance.

The superiority of the lidocaine lozenge may be because of the longer contact time in the mouth and, therefore, better distribution and penetration of lidocaine into the pharyngeal mucosa. The continuous release of lidocaine from sucking on a lozenge, in addition to swallowing the saliva mixed with the local anesthetic, allows a homogenous and slow spread of this anesthetic. This provides ample time for it to exert its anesthetic effect, not only to the pharyngeal mucosa, but also to the soft palatal and posterior third of the tongue - the area containing the deep pressure receptors responsible for the gag reflex. ${ }^{10}$

There was no significant difference between the numbers of patients who received sedation in the two groups. A reason for this could be that most patients received sedation before the UGE started; therefore, the patients did not have the opportunity to evaluate the effect of the topical anesthesia. The nonsedated patients' results showed sedation did not influence the patients' evaluation.

The lidocaine dose used in this study was $100 \mathrm{mg}$ because it is common practice at Hvidovre University Hospital to administer this dose as the viscous oral solution before UGE. The effectiveness of topical anesthesia is probably related to the lidocaine dosage. Other studies showed that $100 \mathrm{mg}$ of lidocaine is an effective dose administered before an UGE. ${ }^{9,12}$

The results showed the lozenge was significantly better in taste and texture compared with the solution, and indicate the bitter taste of lidocaine can be successfully disguised with liquorice flavor and aspartame. Since the patients who received the lozenge had a higher acceptance of the UGE, the better taste and texture of the lozenge may have had an impact on patient acceptance.

The present study has some limitations. To improve the study design, the patients should have been offered sedation only after the local anesthetic was administered, or only nonsedated patients should have been included, because their assessments would not have been affected by the sedation. Another impact on the results could be the high number of endoscopists performing the procedures in the study. The result of the endoscopists' assessments might have been more uniform if fewer endoscopists had performed the UGEs. However, only experienced endoscopists performed the procedures.

\section{Conclusion}

The results indicate the lozenge improved patient acceptance of the gag reflex during UGE, and the lozenge was effective and well accepted as local anesthesia because of the palatable taste and texture. These promising results suggest the lozenge can reduce patient discomfort during UGE, thereby improving patient acceptance of the procedure. If integrated in the UGE procedure, the lozenge could reduce the severe discomfort during UGE experienced by many patients worldwide.

\section{Disclosure}

The authors have no conflicts of interest or financial ties to disclose.

\section{References}

1. Leslie K, Stonell CA. Anaesthesia and sedation for gastrointestinal endoscopy. Curr Opin Anaesthesiol. 2005;18:431-436.

2. Campo R, Brullet E, Montserrat A, et al. Identification of factors that influence tolerance of upper gastrointestinal endoscopy. Eur $J$ Gastroenterol Hepatol. 1999;11:201-204.

3. Shaoul R, Higaze H, Lavy A. Evaluation of topical pharyngeal anaesthesia by benzocaine lozenge for upper endoscopy. Aliment Pharmacol Ther. 2006;24:687-694.

4. Asante MA, Northfield TC. Variation in taste of topical lignocaine anaesthesia for gastroscopy. Aliment Pharmacol Ther. 1998;12:685-686.

5. Chan CK, Fok KL, Poon CM. Flavored anesthetic lozenge versus Xylocaine spray used as topical pharyngeal anesthesia for unsedated esophagogastroduodenoscopy: a randomized placebo-controlled trial. Surg Endosc. 2009;24:897-901.

6. Heuberger S, Weber KB, Sonnenberg A, et al. Topical anesthesia in preendoscopic medication: spray versus lozenges. Endoscopy. 1979;11: 131-132.

7. Isenberg G. Topical anesthesia: to use or not to use - that is the question. Gastrointest Endosc. 2001;53:130-133.

8. Aronson J. Meyler's Side Effect of Drugs Used in Anesthesia. Oxford: Elsevier Science and Technology; 2009.

9. Mulcahy HE, Greaves RR, Ballinger A, et al. A double-blind randomized trial of low-dose versus high-dose topical anaesthesia in unsedated upper gastrointestinal endoscopy. Aliment Pharmacol Ther. 1996;10:975-979.

10. Ayoub C, Skoury A, Abdul-Baki H, Nasr V, Soweid A. Lidocaine lollipop as single-agent anesthesia in upper GI endoscopy. Gastrointest Endosc. 2007;66:786-793.

11. Amornyotin S, Srikureja W, Chalayonnavin W, Kongphlay S, Chatchawankitkul S. Topical viscous lidocaine solution versus lidocaine spray for pharyngeal anesthesia in unsedated esophagogastroduodeno scopy. Endoscopy. 2009;41:581-586.

12. Jameson JS, Kapadia SA, Polson RJ, McCarthy PT, Misiewicz JJ. Is oropharyngeal anaesthesia with topical lignocaine useful in upper gastrointestinal endoscopy? Aliment Pharmacol Ther. 1992;6: 739-744. 


\section{Publish your work in this journal}

Local and Regional Anesthesia is an international, peer-reviewed, open access journal publishing on the development, pharmacology, delivery and targeting and clinical use of local and regional anesthetics and analgesics. The journal welcomes submitted papers covering original research, basic science, clinical studies, reviews \& evaluations,

guidelines, expert opinion and commentary, case reports and extended reports. The manuscript management system is completely online and includes a very quick and fair peer-review system, which is all easy to use. Visit http://www.dovepress.com/testimonials.php to read real quotes from published authors.

Submit your manuscript here: http://www.dovepress.com/local-and-regional-anesthesia-journal 